\title{
¿Existe relación entre la enfermedad de Alzheimer y defectos en el ADN mitocondrial?
}

\author{
B. Rodríguez-Santiago ${ }^{\text {a }}$ J. Casademont ${ }^{\mathrm{b}}$, V. Nunes $^{\mathrm{a}}$
}

IS THERE A RELATION BETWEEN ALZHEIMER'S DISEASE AND DEFECTS OF MITOCHONDRIAL DNA?

\begin{abstract}
Summary. Introduction. Various studies have related Alzheimer's disease (AD) with mitochrondrial defects. These defects may be structural, biochemical or genetic in type. Amongst the genetic defects the rearrangement and particular mutations described in mitochondrial DNA ( mtDNA) are striking. Objective. To study the incidence of rearrangement and 4 particular mutations in the mtDNA of patients with $A D$, and determine the possible differences from persons taken as controls. Patients and methods. Necropsies of the cerebellum, frontal cortex and hippocampus of patients with AD and controls. We also used blood from living patients diagnosed as having EA and from controls. The samples were analysed using hybrid Southern blot with a mitochondrial probe. Particular mutations G3196A, A3397G, A4336G and G5460A/T were also analysed. Results. No differences were found between the patients and controls, in either brain tissue or blood on analysis using Southern. No association was found between the particular mutations analysed and the AD of our samples. Conclusions. The results obtained did not support the hypothesis of involvement of mitochondria in AD. Regarding rearrangement and the 4 particular mutations analysed in the mtDNA of our samples. However, this does not rule out the possible existence of other particular mutations which were not analysed and/or other mitochondrial defects which contribute to the development of AD. [REV NEUROL 2001; 33: 301-5]

Key words. Alzheimer's disease. Deletions. Mitochondria. Mitochondrial DNA. Particular mutations. Rearrangements. Respiratory chain.
\end{abstract}

\section{INTRODUCCIÓN}

La enfermedad de Alzheimer (EA) es una de las formas más comunes de demencia senil y presenil. Se caracteriza por un progresivo declive cognitivo global y del comportamiento y se define histológicamente por la presencia de placas amiloides depósitos extracelulares que consisten principalmente en agregaciones de péptido $\beta$-amiloide-y de los denominados ovillos neurofibrilares -depósitos intracelulares mayoritariamente formados por proteína tau hiperfosforilada- [1]. Las causas genéticas de la enfermedad parecen ser heterogéneas. La EA de inicio temprano puede asociarse con mutaciones en loci de los cromosomas 1, 14 y 21 [2-5]. La EA de inicio tardío parece diferir genéticamente de la temprana; un factor de riesgo identificado para la EA tardía es la presencia del alelo 4 de la apolipoproteína $\mathrm{E}[6,7]$.

Diferentes estudios indican que existen factores genéticos que desempeñan un papel importante en la etiología de la EA $[8,9]$. La incidencia de la enfermedad es mayor en mujeres [10], aunque este efecto puede ser consecuencia de su mayor longevidad relativa. El genoma mitocondrial es una molécula circular

Recibido:08.02.01. Aceptadotras revisión externa sinmodificaciones: 09.04.01.

${ }^{a}$ Centro de Genética Médica y Molecular-IRO. ${ }^{b}$ Grupo de Investigación Muscular. Hospital Clínic. IDIBAPS. Universitat de Barcelona. Barcelona, España.

Correspondencia: Dra. Virginia Nunes. Centro de Genética Médica y Molecular-IRO. Hospital Duran i Reynals. Autovía de Castelldefels, $\mathrm{km}$ 2,7. E-08907 L'Hospitalet de Ll., Barcelona. Fax: +3493 2607776. E-mail: vnunes@iro.es

Agradecimientos. A los pacientes con EA y a los individuos que han participado en el estudio. A Pilar Viedma y Rafael Blesa, del Servicio de Neurología del Hospital Clínic de Barcelona, por facilitar las muestras. A Montse Gómez y Enric Doménech, por sus comentarios. Este trabajo contó con las ayudas de la Fundació La Marató de TV3 no 2002/97 y 2102/ 97 (V. Nunes y J. Casademont) y de la CICYT: SAF 99-0079 a V. Nunes. Benjamín Rodríguez-Santiago es becario predoctoral de la Fundació La Marató de TV3.

(C) 2001, REVISTA DE NEUROLOGÍA de doble cadena de ADN de 16,5 kilobases (kb), que se transmite exclusivamente a través de la línea materna. En los últimos años se han descrito diversas anomalías en el metabolismo mitocondrial asociadas a la EA [11-26]. Estos defectos en las mitocondrias incluyen alteraciones de tipo estructural [11], bioquímico [15-17] y genético [16-22]. Entre los defectos estructurales destacan los cambios observados en las mitocondrias de líneas celulares de cíbridos, que afectan a las membranas y a la matriz [11]. También se han observado fallos en el control homeostático del calcio [12], que a su vez está relacionado con la apoptosis $[13,14]$, aumento en la presencia de radicales libres y especies reactivas del oxígeno que causan daño en el ADN mitocondrial (ADNmt), el ADN nuclear y las membranas [8,14]. La disfunción bioquímica se ha relacionado con una disminución en la actividad de la cadena respiratoria [15], especialmente en la citocromo c oxidasa [16]. Esto conlleva un desajuste en el metabolismo mitocondrial oxidativo [17]. El cerebro presenta una gran dependencia metabólica de la producción de ATP a través de la fosforilación oxidativa. En tejido cerebral de pacientes con EA se ha demostrado un desacoplamiento parcial de la fosforilación oxidativa [17]. En el aspecto genético se han observado variaciones en las proporciones de moléculas de ADNmt con la deleción común -tamaño de 4,9kb-, según el área cerebral analizada [18,19], así como la presencia de mutaciones puntuales [20-23]. También se ha descrito una reducción en la expresión de algunos genes mitocondriales [24].

Las mutaciones puntuales y reordenamientos en el ADNmt constituyen atractivos candidatos para explicar algunos de los defectos hallados en la función mitocondrial. Mutaciones en el genoma mitocondrial pueden causar defectos graves en la cadena respiratoria y provocar una serie de fenotipos degenerativos graves, como sordera, ataxia, cardiomiopatía y demencia de tipo Alzheimer [27]. En diversos tejidos, entre ellos el cerebro, se ha observado que con la edad se produce un aumento de las mutaciones somáticas y de deleciones en el ADNmt [28,29]. Si estas mutaciones y deleciones alcanzan o no niveles suficientes para 
tener efectos negativos en la célula aún no se ha podido confirmar claramente - dada la convivencia entre ADNmt normales y ADNmt mutados en una misma célula, debe haber un umbral a partir del cual los ADNmt mutados pueden provocar disfunciones en la mitocondria-, pero su acumulación se encuentra acelerada en el tejido cerebral de pacientes con EA [19]. Sin embargo, existen otros trabajos en los que no se han hallado pruebas de esta asociación entre la mitocondria y EA, cuestionando la relevancia de estas anomalías en el desarrollo de la enfermedad [30-35].

Como consecuencia de la disparidad en los resultados observados en la bibliografía, en lo referente a reordenamientos y mutaciones puntuales, decidimos evaluar el estado del ADNmt en muestras de varias regiones del cerebro (cerebelo, hipocampo, córtex frontal) y en sangre de pacientes con EA y de controles. Se estudió la incidencia de cuatro mutaciones puntuales distintas previamente asociadas a la EA: las variantes G3196A, A3397G, A4336G y G5460A/T [21-23] -localizadas en los genes ND1, 16 SrARN, tARN ${ }^{\mathrm{Gln}}$ y ND2, respectivamente-, mediante amplificación por PCR (reacción en cadena de la polimerasa) y digestión con enzimas de restricción. La existencia de reordenamientos en el ADNmt (deleciones y duplicaciones) se analizó mediante Southern blot e hibridación.

\section{PACIENTES Y MÉTODOS Muestras}

Para el estudio se utilizaron muestras de cerebelo y córtex frontal de 12 pacientes afectos de EA y siete individuos control. También se obtuvieron muestras de hipocampo en 10 de los pacientes y cuatro de los controles. Estas muestras las proporcionó el Banco de Tejidos Neurológicos del Hospital Clínic-Universitat de Barcelona. En todos los casos se realizó un análisis histopatológico para confirmar la presencia de depósitos de proteína $\beta$-amiloide, que definen la EA. Las muestras control pertenecían a individuos sin demencia u otra enfermedad neurológica en los que, además, los análisis neuropatológicos excluyeron la presencia de placas seniles u ovillos neurofibrilares. En todas las muestras de tejido cerebral, el tiempo de congelación después de la muerte fue inferior a las 12 horas. El intervalo de edades fue 56-84 años en los pacientes de EA y 55-70 años en los individuos control. Además, se recogieron y analizaron muestras de sangre de 17 pacientes de EA vivos -intervalo de edades, 59-87-, diagnosticados mediante criterios comúnmente aceptados [36], y de 27 individuos control sin historial de enfermedad neurológica ni demencia -intervalo de edades, 58-94.

Todas las muestras de Alzheimer correspondían a formas esporádicas de la enfermedad. El consentimiento para el estudio se obtuvo de acuerdo con las normas del comité ético del centro, siguiendo la declaración de Helsinki.

\section{Análisis del ADNmt}

\section{Preparación del ADN}

Se procesaron $50 \mathrm{mg}$, aproximadamente, de cada muestra de región cerebral congelada y $15 \mathrm{ml}$ de las muestras de sangre para la extracción de ADN total (genómico y mitocondrial), según procedimientos estándar modificados [37].

\section{Análisis moleculares}

Se digirieron $4 \mu \mathrm{g}$ de ADN total de cada muestra con el enzima de restricción $P v u$ II (Gibco). Esta enzima corta el ADNmt por un solo punto y lo alinea. El ADN digerido se sometió a electroforesis en gel al 0,8\% de agarosa y se transfirió a una membrana de nilón (Amersham), según el método de Ausubel et al [37].

Como sonda se utilizó ADNmt total a una concentración de $50 \mathrm{ng} / \mu \mathrm{l}$ marcado con $\alpha{ }_{-}^{32} \mathrm{P}(\mathrm{dCTP})$, mediante la técnica de cebado aleatorio (del inglés, random priming) [38]. La hibridación del filtro con el ADNmt humano marcado y los subsiguientes lavados se llevaron a cabo siguiendo el protocolo descrito por el fabricante de la membrana, y se procedió a continuación a la autorradiografía del mismo.

\section{PCR y análisis de las mutaciones puntuales}

Cada mutación se analizó mediante amplificación por PCR de la región del ADNmt susceptible de presentar la mutación, seguida de una digestión con el enzima de restricción adecuado, tal y como se describe a continuación:

- Mutaciones G3196A y A3397G. A partir de 200 ng de ADN se amplificó un fragmento de $474 \mathrm{pb}$ que contenía parte del gen ND1 y del gen 16SrRNA, bajo las siguientes condiciones: oligonucleótidos L·leu-1 -posición en la cadena light (L) del ADNmt 3164-3183- y H363 -nucleótidos (nt) 3618-3637 de la cadena heavy $(\mathrm{H})$ a $1 \mathrm{mM}$, dNTPs a $200 \mathrm{mM}$ y 1,5 unidades de Taq (Roche). Las condiciones de PCR fueron: 5 min a $95^{\circ} \mathrm{C}$, seguido de 35 ciclos compuestos de tres segmentos $-35 \mathrm{~s}$ a $94{ }^{\circ} \mathrm{C}, 35 \mathrm{~s}$ a $56{ }^{\circ} \mathrm{C}$ y $35 \mathrm{~s} \mathrm{a} 72{ }^{\circ} \mathrm{C}-$, más una extensión final de $10 \mathrm{~min}$ a $72{ }^{\circ} \mathrm{C}$. Una alícuota de la reacción se digirió con el enzima Dde I (Roche) para la mutación G3196A o con Rsa I (New England Biolabs) para la A3397G; los fragmentos se separaron en geles de acrilamida al 7\%. La mutación A3397G crea una diana Rsa I, mientras que la G3196A anula una diana Dde I.

- Mutación A4336G. Un fragmento del gen tARN ${ }^{\mathrm{Gln}}$ de 785 pb se amplificó de forma similar a lo explicado anteriormente, usando los oligonucleótidos L401 (nt 4013-4031) y H479 (nt 4797-4778). Las condiciones de PCR fueron: 5 min a $95{ }^{\circ} \mathrm{C}, 35$ ciclos compuestos por $35 \mathrm{~s} \mathrm{a} 94{ }^{\circ} \mathrm{C}$, $35 \mathrm{~s}$ a $51^{\circ} \mathrm{C}, 35 \mathrm{~s} \mathrm{a} 72^{\circ} \mathrm{C}$, y finalmente una extensión de 10 min a $72{ }^{\circ} \mathrm{C}$. La digestión se realizó con el enzima Nla III (New England Biolabs) y la electroforesis en un gel de acrilamida al 6\%. La mutación A4336G crea una diana.

- Mutación G5460A/T. Los cambios en la posición 5460 se detectaron usando los oligonucleótidos L2 (nt 5440-5459) y H2 (nt 5898-5879) con las condiciones de PCR: 5 min a $95^{\circ} \mathrm{C}, 35$ ciclos compuestos por $35 \mathrm{~s}$ a $94{ }^{\circ} \mathrm{C}, 35 \mathrm{~s}$ a $59^{\circ} \mathrm{C}, 35 \mathrm{~s} \mathrm{a} 72{ }^{\circ} \mathrm{C}$, y una extensión final de $10 \mathrm{~min}$ a $72{ }^{\circ} \mathrm{C}$. L2 es un oligonucleótido de mutagénesis: en la posición que corresponde al nt 5458 de la cadena L del ADNmt se ha sustituido el nt normal (una T) por una G. Este cambio crea una diana Hha I (Amersham) en moléculas salvajes. Una A o una T en la posición 5460 anula esta diana; además, una T crea una diana para el enzima Alu I (Amersham).

\section{Secuenciación}

Para identificar los cambios en los patrones de bandas anómalos que no correspondían a las mutaciones a estudiar se realizó un análisis de secuencia: el producto de PCR del fragmento con patrón irregular se purificó mediante columna (Qiagen). La PCR de secuencia se realizó con el equipo ABI Prism Big Dye Terminator Cycle Sequencing Ready Reaction (Applied Biosyste$\mathrm{ms}$ ), siguiendo las recomendaciones del fabricante. Los resultados se recogieron y analizaron con los programas de ordenador del equipo ABI Prism 377 DNA Sequencer (Applied Biosystems) y el programa Sequencher (Gene Codes Corporation).

\section{RESULTADOS}

Los análisis mediante Southern blot e hibridación de los ADNmt obtenidos de necropsias cerebrales y de sangre de casos de Alzheimer no mostraron presencia de deleciones u otros reordenamientos del ADNmt, tales como dimerizaciones o duplicaciones. En la figura se muestra una imagen típica del patrón encontrado. El ADN de las muestras, hibridado contra una sonda específica de ADNmt total, puso de manifiesto en todos los casos de EA la existencia de moléculas con una banda de $16,5 \mathrm{~kb}$ de tamaño, correspondiente a una población de moléculas de ADNmt íntegras. Para un mismo individuo tampoco se observaron diferencias en el patrón obtenido mediante Southern blot, en las muestras correspondientes a las diferentes regiones cerebrales analizadas.

Los resultados obtenidos en el estudio de mutaciones puntuales se encuentran resumidos en la tabla. Las mutaciones G3196A, A3397G y G5460A/T no estaban presentes en ninguna muestra, ya sea de tejidos o muestras de sangre, ni en pacientes o controles. La mutación A4336G se detectó en todas las regiones cerebrales de dos casos con EA y no apareció en ningún control de muestras de tejido. En muestras de sangre, la mutación A4336G apareció en un control, pero no se observó en ningún paciente.

Se detectaron patrones anómalos que no correspondían con las mutaciones estudiadas. Los cambios encontrados fueron los siguientes: el cambio A4136G se detectó en linfocitos de un control, en las tres regiones cerebrales de un control y de un paciente con EA; el cambio T4216C se observó en los 
Pacientes

Controles

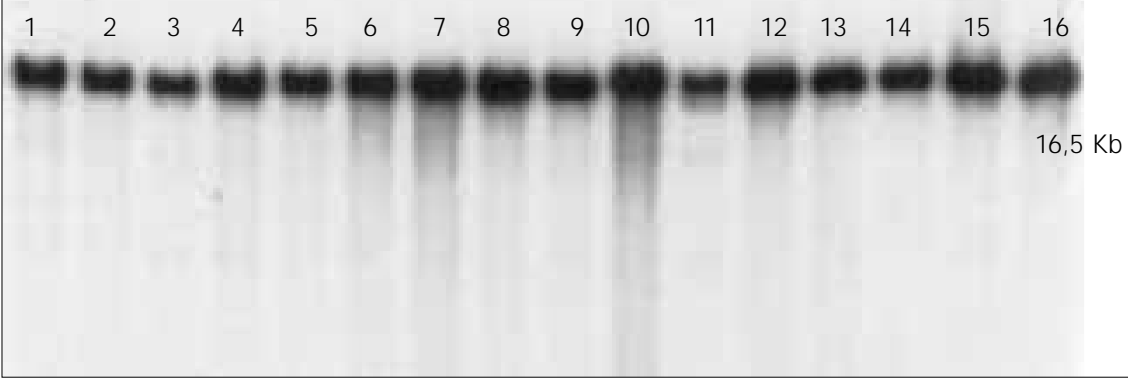

Figura. Ejemplo de imagen de southem de ADN extraído a partir de necropsias de cerebelo, digeridos con Pvu II e hibridados con una sonda de ADNmt total. En los southem de muestras de las otras regiones y de sangre se obtuvieron imágenes similares, con una banda única de $16,5 \mathrm{~kb}$, correspondiente a ADNmt íntegro (camiles 1-9: pacientes con EA; carriles 10-16: controles).

Tabla. Frecuencia de mutaciones en el ADNmt de pacientes con EA y de controles.

\begin{tabular}{llcccccccc}
\hline $\begin{array}{l}\text { Gen } \\
\text { ADNmt }\end{array}$ & Variante & \multicolumn{2}{c}{ Cerebelo } & \multicolumn{2}{c}{ Córtex frontal } & Hipocampo & \multicolumn{2}{c}{ Sangre } \\
\hline & & C & P & C & P & C & P & C & P \\
\hline 16SrARN & G3196A & $0 / 7$ & $0 / 12$ & $0 / 7$ & $0 / 12$ & $0 / 4$ & $0 / 10$ & $0 / 27$ & $0 / 17$ \\
\hline ND1 & A3397G & $0 / 7$ & $0 / 12$ & $0 / 7$ & $0 / 12$ & $0 / 4$ & $0 / 10$ & $0 / 27$ & $0 / 17$ \\
\hline tARNGIn & A4336G & $0 / 7$ & $2 / 12$ & $0 / 7$ & $2 / 12$ & $0 / 4$ & $1 / 10$ & $1 / 27$ & $0 / 17$ \\
\hline ND2 & G5460A/T & $0 / 7$ & $0 / 12$ & $0 / 7$ & $0 / 12$ & $0 / 4$ & $0 / 10$ & $0 / 27$ & $0 / 17$ \\
\hline
\end{tabular}

C: controles; P: pacientes.

linfocitos de un control y de cuatro pacientes con EA, y también en las tres regiones de un caso con EA; por último, se detectaron el cambio G3423T en las tres regiones de seis individuos con EA y el cambio G4580A en otro individuo.

\section{DISCUSIÓN}

El presente estudio se realizó con el objetivo de observar el estado del ADNmt en cuanto a la posible presencia de mutaciones puntuales y reordenamientos en el tejido principalmente afectado en la EA, el cerebro. Para ello, se analizaron necropsias de cerebelo, hipocampo y córtex frontal tomadas de enfermos y se compararon con necropsias de individuos control.

La implicación mitocondrial en el desarrollo de la EA ha motivado diversas controversias. Se han descrito numerosas alteraciones en la función mitocondrial, pero aún está por aclarar si esas alteraciones desempeñan un papel primario o son un hecho secundario en la etiología de la enfermedad. Por tratarse de una enfermedad neurodegenerativa y progresiva, de años de duración, es fácil pensar que los defectos hallados en el metabolismo mitocondrial podrían ser consecuencia del progresivo deterioro al que se someten las principales áreas cerebrales afectadas. Diversos estudios han demostrado la presencia de factores perjudiciales para el ADNmt en enfermos de Alzheimer. Estos factores suponen un entorno potencialmente dañino para el metabolismo de la mitocondria: la presencia de proteína amiloide genera radicales libres y estrés oxidativo, con repercusiones sobre la cadena respiratoria y el ADNmt. A esto hay que añadir la práctica ausencia de mecanismos de reparación del ADNmt. Es por ello que resulta sorprendente no encontrar en las muestras analizadas -con confirmación histopatológica de EA- mayor presencia de mutaciones puntuales y reordenamientos en el ADNmt. No se puede descartar la existencia de moléculas delecionadas en cantidad inferior al 5\% -nivel de resolución de la hibridación con sonda radioactiva. En cualquier caso, una proporción de este orden sería insuficiente para producir una disfunción de la mitocondria. Como se observa en la figura, la banda de $16,5 \mathrm{~kb}$ correspondiente a una población normal es muy abundante en todos los pacientes y muy similar a la de los individuos control. La cantidad de deleciones halladas en otros estudios varía según la zona cerebral analizada, siendo las áreas del córtex las más afectadas. En nuestro estudio, las tres regiones estudiadas no mostraron diferencias para un mismo individuo.

Trabajos previos han relacionado las mutaciones puntuales G3196A, A3397G, A4336G y G5460A/T con la EA [21-23]. Sin embargo, otros grupos no han encontrado esta relación [32-35]. Los datos de nuestro estudio tampoco aportan pruebas que apoyen la implicación de estas mutaciones en la enfermedad. No detectamos esas mutaciones en las muestras analizadas, con la excepción de la A4336G, descubierta en todas las áreas cerebrales de dos casos de EA. Esto parece sugerir que esta mutación puntual puede desempeñar algún papel en el cerebro de enfermos de Alzheimer. Para comprobar la relevancia de esta mutación en las áreas cerebrales sería preciso analizar más muestras, ya que los datos obtenidos no permiten establecer una significación estadística. Por otra parte, el hecho de hallar esta mutación en sangre de un control cuestiona su papel en la enfermedad y, eventualmente, podría tratarse de un polimorfismo. Como sugieren estudios previos [39], el cambio A4336G podría ser un marcador de la implicación del ADNmt en algunos casos de EA, pero es improbable que tenga un papel significativo en la mayoría de pacientes.

Aunque la sangre no es un tejido diana de la enfermedad y los defectos hallados en linfocitos pueden tener una importancia menor -si posteriormente no se confirman en tejido neurológico-, el hecho de que se hayan descrito algunas alteraciones mitocondriales en sangre de individuos con EA [25,26], unido a la posibilidad de disponer de muestras de pacientes vivos y a la idea de hallar un marcador para discriminar rápidamente a los pacientes, nos llevó a realizar los estudios con este tipo de muestras. Definitivamente, nuestros resultados no han permitido identificar ningún tipo de marcador para la enfermedad. Hay que indicar que las bandas observadas mediante hibridación correspondientes a ADNmt obtenido de sangre son más débiles que las procedentes de áreas de cerebro. Esto se explica sencillamente por la mayor presencia de mitocondrias en tejidos posmitóticos, como son el cerebro o el músculo esquelético, que presentan una mayor demanda energética de ATP. 
Los cambios A4136G y T4216C encontrados-localizados en el gen mitocondrial ND1- se clasifican como polimorfismos en gran parte de la literatura, aunque algunos los consideran mutacionesLHON (del inglés, Leber's Hereditary Optic Neuropathy) de tipo secundario. Los cambios encontrados en las posiciones 3423 (gen mtND1) y 4580 (gen mtND2) se han descrito previamente como polimorfismos.

Los datos del presente artículo no permiten afirmar que en nuestras muestras con EA exista una alteración del genoma mitocondrial, en cuanto a la presencia de reordenamientos y de estas cuatro mutaciones puntuales. No obstante, otro estudio realizado con las mismas muestras apuntaba la posibilidad de que existieran diferencias en la cantidad de ADNmt (depleción), especialmente en la región del córtex frontal; en ésta, los pacientes de EA presentaban una reducción de hasta un $28 \%$ en su contenido de ADNmt, respecto a los controles [40]. Si las alteraciones descritas hasta el momento son primarias (patogénicas) o secundarias -consecuencia de la degeneración neuronal-, está por dilucidar. El estudio más exhaustivo de los defectos del ADNmt, junto a otros aspectos del metabolismo mitocondrial, incluida su relación con el genoma nuclear, debe servir para aclarar el papel que desempeñan las mitocondrias en las enfermedades neurodegenerativas y, en concreto, en la EA. En cualquier caso, el detenido conocimiento del estado mitocondrial deberá ayudar a comprender el delicado equilibrio que regula la pérdida selectiva de neuronas en la EA.

\section{BIBLIOGRAFÍA}

1. Dickson DW. The pathogenesis of senile plaques. J Neuropathol Exp Neurol 1997; 56: 321-39.

2. Chartier-Harlin MC, Crawford F, Houlden H, Warren A, Hughes D, Fidani L, et al. Early-onset Alzheimer's disease caused by mutations at codon 717 of the beta-amyloid precursor protein gene. Nature 1991; 353: 844-6.

3. Goate A, Chartier-Harlin MC, Mullan M, Brown J, Crawford F, Fidani $\mathrm{L}$, et al. Segregation of a missense mutation in the amyloid precursor protein gene with familial Alzheimer's disease. Nature 1991; 349: 704-10.

4. Levy-Lahad E, Wasco W, Poorkaj P, Romano DM, Oshima J, Pettingell WH, et al. Candidate gene for the chromosome 1 familial Alzheimer's disease locus. Science 1995; 269: 973-7.

5. Sherrington R, Rogaev EI, Liang Y, Rogaeva EA, Levesque G, Ikeda $\mathrm{M}$, et al. Cloning of a gene bearing missense mutations in early-onset familial Alzheimer's disease. Nature 1995; 375: 754-60.

6. Corder EH, Saunders AM, Strittmatter WJ, Schmechel DE, Gaskell PC, Small GW, et al. Gene dose of apolipoprotein E type 4 allele and the risk of Alzheimer's disease in late onset families. Science 1993; 261: 921-3.

7. Strittmatter WJ, Saunders AM, Schmechel D, Pericak-Vance M, Enghild J, Salvesen GS, et al. Apolipoprotein E: high-avidity binding to beta-amyloid an increased frequency of type 4 allele in late-onset familial Alzheimer disease. Proc Natl Acad Sci U S A 1993; 90: 1977-81.

8. Christen Y. Oxidative stress and Alzheimer disease. Am J Clin Nutr 2000; 71 (Suppl): S621-9.

9. Price DL, Sisodia SS, Borchelt DR. Alzheimer disease-when and why? Nat Genet 1998; 19: 314-6.

10. Edland SD, Silverman JM, Peskind ER, Tsuang D, Wijsman E, Morris JC. Increased risk of dementia in mothers of Alzheimer's disease cases: evidence for maternal inheritance. Neurology 1996; 47: 254-6.

11. Trimmer PA, Swerdlow RH, Parks JK, Keeney P, Bennett JP Jr, Miller SW, et al. Abnormal mitochondrial morphology in sporadic Parkinson's and Alzheimer's disease cybrid cell lines. Exp Neurol 2000; 162: 37-50.

12. Sheehan JP, Swerdlow RH, Miller SW, Davis RE, Parks JK, Parker $\mathrm{WD}$, et al. Calcium homeostasis and reactive oxygen species production in cells transformed by mitochondria from individuals with sporadic Alzheimer's disease. J Neurosci 1997; 17: 4612-22.

13. Suzuki A, Tsutomi Y, Yamamoto N, Shibutani T, Akahane K. Mitochondrial regulation of cell death: mitochondria are essential for procaspase 3-p21 complex formation to resist Fas-mediated cell death. Mol Cell Biol 1999; 19: 3842-7.

14. Deng G, Su JH, Ivins KJ, van Houten B, Cotman CW. Bcl-2 facilitates recovery from DNA damage after oxidative stress. Exp Neurol 1999; 159: 309-18.

15. Parker WD Jr, Parks J, Filley CM, Kleinschmidt-DeMasters BK. Electron transport chain defects in Alzheimer's disease brain. Neurology 1994; 44: 1090-6.

16. Mutisya EM, Bowling AC, Beal MF. Cortical cytochrome oxidase activity is reduced in Alzheimer's disease. J Neurochem 1994; 63: 2179-84.

17. Redjems-Bennani N, Jeandel C, Lefebvre E, Blain H, Vidailhet M, Gueant $\mathrm{JL}$. Abnormal substrate levels that depend upon mitochondrial function in cerebrospinal fluid from Alzheimer patients. Gerontology 1998; 44: 300-4.

18. Hamblet NS, Castora FJ. Elevated levels of the Kearns-Sayre syndrome mitochondrial DNA deletion in temporal cortex of Alzheimer's patients. Mutat Res 1997; 379: 253-62.

19. Corral-Debrinski M, Horton T, Lott MTDNA, et al. Marked changes in mitochondrial DNA deletion levels in Alzheimer brains. Genomics 1994; 23: 471-6.

20. Lin FH, Lin R, Wisniewski HM, Hwang YW, Grundke-Iqbal I, Healy-Louie G, et al. Detection of point mutations in codon 331 of mitochondrial NADH dehydrogenase subunit 2 in Alzheimer's brains. Biochem Biophys Res Commun 1992; 182: 238-46.

21. Shoffner JM, Brown MD, Torroni A, Lott MT, Cabell MF, Mirra SS, et al. Mitochondrial DNA variants observed in Alzheimer disease and Parkinson disease patients. Genomics 1993; 17: 171-84.

22. Hutchin T, Cortopassi G. A mitochondrial DNA clone is associated with increased risk for Alzheimer disease. Proc Natl Acad Sci U S A 1995; 92: 6892-5.

23. Egensperger R, Kosel S, Schnopp NM, Mehraein P, Graeber MB. Association of the mitochondrial tRNA(A4336G) mutation with Alzheimer's and Parkinson's diseases. Neuropathol Appl Neurobiol 1997; 23: 315-21.

24. Aksenov MY, Tucker HM, Nair P, Aksenova MV, Butterfield DA, Estus $\mathrm{S}$, et al. The expression of several mitochondrial and nuclear genes encoding the subunits of electron transport chain enzyme complexes, cytochrome c oxidase, and NADH dehydrogenase, in different brain regions in Alzheimer's disease. Neurochem Res 1999; 24: 767-74.

25. Parker WD Jr. Cytochrome oxidase deficiency in Alzheimer's disease. Ann N Y Acad Sci 1991; 640: 59-64.

26. Mecocci P, Polidori MC, Ingegni T, Cherubini A, Chionne F, Cecchetti $\mathrm{R}$, et al. Oxidative damage to mtDNA in lymphocytes from $\mathrm{AD}$ patients. Neurology 1998; 51: 1014-7.

27. DiMauro S, Wallace DC. Mitochondrial DNA in Human Pathology New York: Raven Press; 1993.

28. Soong NW, Hinton DR, Cortopassi G, Arnheim N. Mosaicism for a specific somatic mitochondrial DNA mutation in adult human brain. Nat Genet 1992; 2: 318-23.

29. Corral-Debrinski M, Horton T, Lott MT, Shoffner JM, Beal MF, Wallace DC. Mitochondrial DNA deletions in human brain: regional variability and increase with advanced age. Nat Genet 1992; 2: 324-9.

30. Lezza AM, Mecocci P, Cormio A, Beal MF, Cherubini A, Cantatore $\mathrm{P}$, et al. Mitochondrial DNA $4977 \mathrm{bp}$ deletion and OH8dG levels correlate in the brain of aged subjects but not Alzheimer's disease patients. FASEB J 1999; 13: 1083-8.

31. Blanchard BJ, Park T, Fripp WJ, Lerman LS, Ingram VM. A mitochondrial DNA deletion in normally aging and in Alzheimer brain tissue. Neuroreport 1993; 4: 799-802.

32. Janetzky B, Schmid C, Bischof F, Frolich L, Gsell W, Kalaria RN, et al. Investigations on the point mutations at nt 5460 of the mtDNA in different neurodegenerative and neuromuscular diseases. Eur Neurol 1996; 36: 149-53.

33. Kosel S, Egensperger R, Mehraein P, Graeber MB. No association of mutations at nucleotide 5460 of mitochondrial NADH dehydrogenase with Alzheimer's disease. Biochem Biophys Res Commun 1994; 203: 745-9.

34. Wragg MA, Talbot CJ, Morris JC, Lendon CL, Goate AM. No association found between Alzheimer's disease and a mitochondrial tRNA glutamine gene variant. Neurosci Lett 1995; 201: 107-10.

35. Hutchin TP, Heath PR, Pearson RC, Sinclair AJ. Mitochondrial DNA mutations in Alzheimer's disease. Biochem Biophys Res Commun 1997; 241: 221-5.

36. McKhann G, Drachman D, Folstein M, Katzman R, Price D, Stadlan EM Clinical diagnosis of Alzheimer's disease: report of the NINCDS-ADRDA Work Group under the auspices of Department of Health and Human Services Task Force on Alzheimer's Disease. Neurology 1984; 34: 939-44.

37. Ausubel FM, Brent R, Kingston RE, Moore DD, Seidman JG, Smith JA, et al. Current protocols in Molecular Biology. New York: Willey; 1993.

38. Feinberg AP, Vogelstein B. A technique for radiolabeling DNA restriction endonuclease fragments to high specific activity. Anal Biochem 1983; 132: 6-13.

39. Bonilla E, Tanji K, Hirano M, Vu TH, DiMauro S, Schon EA. Mitochondrial involvement in Alzheimer's disease. Biochem Bioph Acta 1999; 1410: 171-82

40. Rodríguez-Santiago B, Casademont J, Nunes V. Is mitochondrial DNA depletion involved in Alzheimer's disease? Eur J Hum Genet 2001; 9: $279-85$. 
¿EXISTE RELACIÓN ENTRE LA ENFERMEDAD DE ALZHEIMER Y DEFECTOS EN EL ADN MITOCONDRIAL?

Resumen. Introducción. Varios estudios han relacionado la enfermedad de Alzheimer (EA) con defectos mitocondriales. Tales defectos incluyen anomalías de tipo estructural, bioquímico y genético. Entre las de tipo genético destacan los reordenamientos y las mutaciones puntuales descritas en el DNA mitocondrial (ADNmt). Objetivo. Estudiar la incidencia de reordenamientos y cuatro mutaciones puntuales en el ADNmt de pacientes con EA y determinar las posibles diferencias respecto a individuos control. Pacientes y métodos. Necropsias de cerebelo, córtex frontal e hipocampo de pacientes con EA y controles. También se dispuso de sangre de enfermos vivos diagnosticados de EA y de controles. Las muestras se analizaron mediante Southern blot, hibridando con una sonda mitocondrial. También se analizaron las mutaciones puntuales G3196A, A3397G, A4336G y G5460A/T. Resultados. No se observaron diferencias entre pacientes y controles, ni en tejidos cerebrales ni en sangre en los análisis realizados mediante southern. No se halló asociación entre las mutaciones puntuales analizadas y la EA en nuestras muestras. Conclusiones. Los resultados obtenidos no apoyan la hipótesis de una implicación mitocondrial en la EA, en cuanto a reordenamientos y las cuatro mutaciones puntuales analizados en el ADNmt en nuestras muestras, lo cual no descarta la posible existencia de otras mutaciones puntuales no analizadas y/u otros defectos mitocondriales que contribuyan al desarrollo de la EA. [REV NEUROL 2001; 33: 301-5] Palabrasclave. Cadena respiratoria. Deleciones. EnfermedaddeAlzheimer. Mitocondria. ADNmt. Mutaciones puntuales. Reordenamientos.
EXISTIRÁ UMA RELAÇÃO ENTRE A DOENÇA

DE ALZHEIMER E OS DEFEITOS NO ADN MITOCONDRIAL?

Resumo. Introdução. Vários estudos relacionaram a doença de Alzheimer (DA) com defeitos mitocondriais. Esses defeitos incluem anomalias de tipo estrutural, bioquímico e genético. Entre as anomalias de tipo genético, destacam-se as reordenações e as mutações pontuais descritas no ADN mitocondrial ( $m t A D N)$. Objectivo. Estudar a incidência de reordenação e 4 mutações pontuais no mtADN em doentes com DA, e determinar as possíveis diferenças em relação aos indivíduos de controlo. Doentes e métodos. Necropsias do cerebelo, córtex frontal e hipocampo de doentes com DA e controlos. Também se dispôs do sangue de doentes vivos diagnosticados com DA, e de controlos. As amostras foram analisadas por Southern blot utilizando uma sonda mitocondrial para hibridar. Foram analisadas as mutações pontuais G3196A, A3397G, A43365G e G5460A/T. Resultados. Não se observaram diferenças entre os doentes e os controlos, nas análises realizadas por Southern, no tecido cerebral, nem no sangue. Não se achou uma associação entre as mutações pontuais analisadas e a DA nas nossas amostras. Conclusões. Os resultados obtidos não sustentam a hipótese de um envolvimento mitocondrial na DA, quanto à reordenação e às 4 mutações pontuais analisadas no mtADN nas nossas amostras, o que não exclui a possível existência de outras mutações pontuais não analisadas elou de outros defeitos mitocondriais que contribuam para o desenvolvimento da DA. [REV NEUROL 2001; 33: 301-5]

Palavras chave. Cadeia respiratória. Delecções. Doença de Alzheimer. Mitocôndria. mtADN. Mutações pontuais. Reordenação. 